\title{
All-ceramic versus titanium-based implant supported restorations: Preliminary 12-months results from a randomized controlled trial
}

\author{
Paul Weigl ${ }^{*}$, Georgia Trimpou², Eleftherios Grizas², Pablo Hess², Georg-Hubertus Nentwig², \\ Hans-Christoph Lauer ${ }^{1}$, Jonas Lorenz ${ }^{1}$ \\ ${ }^{1}$ Department of Prosthodontics, School of Dentistry, Goethe-University Frankfurt am Main, Germany \\ ${ }^{2}$ Department of Oral Surgery and Implantology, School of Dentistry, Goethe-University Frankfurt am Main, Germany
}

\begin{abstract}
PURPOSE. The aim of the present randomized controlled study was to compare prefabricated all-ceramic, anatomically shaped healing abutments followed by all-ceramic abutments and all-ceramic crowns and prefabricated standard-shaped (round-diameter) titanium healing abutments followed by final titanium abutments restored with porcelain-fused-to-metal (PFM) implant crowns in the premolar and molar regions. MATERIALS AND METHODS. Forty-two patients received single implants restored either by all-ceramic restorations (test group, healing abutment, final abutment, and crown all made of zirconia) or conventional titanium-based restorations. Immediately after prosthetic incorporation and after 12 months of loading, implant survival, technical complications, bone loss, sulcus fluid flow rate (SFFR) as well as plaque index (PI) and implant stability (Periotest) were analyzed clinically and radiologically. RESULTS. After 12 months of loading, an implant and prosthetic survival rate of $100 \%$ was observed. Minor prosthetic complications such as chipping of ceramic veneering occurred in both groups. No statistical significant differences were observed between both groups with only a minimum of bone loss, SFFR, and PI. CONCLUSION. All-ceramic implant prostheses including a prefabricated anatomically shaped healing abutment achieved comparable results to titanium-based restorations in the posterior region. However, observational results indicate a benefit as shaping the peri-implant soft-tissue with successive provisional devices and subsequent compression of the soft tissue can be avoided. [J Adv
\end{abstract}

Prosthodont 2019;11:48-54]

KEYWORDS: Ceramic abutments; Titanium abutments; Posterior region; Soft-tissue conditioning; Ceramic sulcus former

\section{INTRODUCTION}

The development and maintenance of a healthy periimplant soft tissue is one of the major goals to achieve in implant dentistry. ${ }^{1-3}$ The simplest, the most common, and

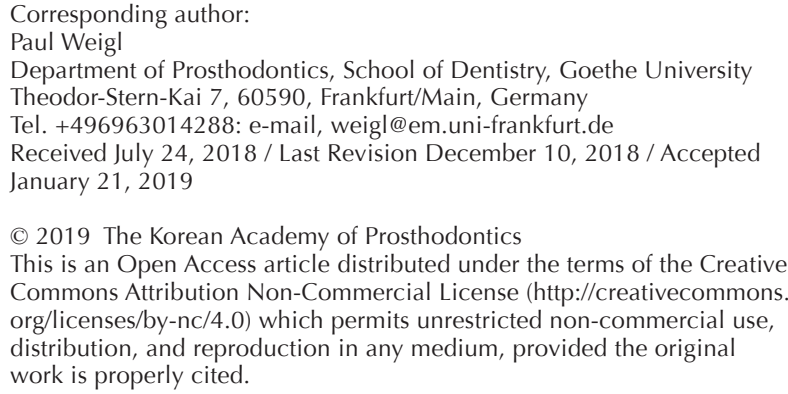

Implant components have been provided free of charge by Dentsply Sirona implants. the most popular way to shape this tissue after osseointegration of the implant is to insert prefabricated healing abutments. These standardized healing abutments are in most cases round-shaped, resulting in emergence profiles dissimilar to those of the tooth being replaced. Initially, healing abutments have been designed in this shape because the cylindrical cross-section of standard (non-individual) abutments makes it easier to align any internal or external indexes. For better shaping of the peri-implant soft-tissue around traditionally shaped abutments, the use of a series of progressively modified provisional crowns is described in the literature. ${ }^{4,5}$ However, this approach is time-consuming and costly to both patient and dentist. Furthermore, the repeated removal and reinsertion of the provisional crowns may cause irritation of the peri-implant soft tissue, as the fragile fibre-attachment may be disconnected and lead to soft-tissue recessions. ${ }^{6}$

Another way to optimize the soft-tissue contours is the 
use of individually customized healing abutments, which are fabricated with computer-aided design and computer-aided manufacturing (CAD/CAM) technology in order to shape the peri-implant soft-tissue anatomically. ${ }^{7}$ However, to optimize the workflow in the dental practice, the healing abutments are needed at the time of implant placement or second-stage surgery. Fabricating CAD/CAM healing abutments requires an impression or an intra-oral scan for the transfer of the implant position and the anatomical situation to the manufacturer. This procedure might also be time-consuming and costly. For this reason, the use of provisional restorations, placed either immediately after implant placement or at the implant second surgery, has been reported to be the most convenient way to shape the periimplant soft tissue. Additionally, the emergence profile can be further adapted and refined by adding PMMA (polymethylmethacrylate) several times to the provisional crowns. ${ }^{8}$

Independent of the types of healing abutment and provisional restoration, customized implant abutments seem to be the choice for definitive prosthetic rehabilitation. ${ }^{9}$ Individualized abutments allow a better control over the design and development of the gingival scalloping and facilitate the removal of cementum remnants..$^{10}$ Compared to gray titanium components, customized zirconia abutments also offer superior aesthetic results because of their toothlike color and influence on the soft- tissue color, especially for patients with thin gingival biotypes. ${ }^{11}$ In the past years, the use of ceramic abutments has been extended to the posterior region, but concerns about the mechanical strength of ceramic abutments are still present. From a scientific point of view, this apparent limitation cannot be supported by scientific data. In contrast, the favourable tissue response to zirconia and patients' preferences for white-coloured materials support the wide indication range of zirconia abutments. ${ }^{12,13}$

The aim of the present randomized controlled trial is to compare prefabricated all-ceramic, anatomically shaped healing abutments followed by all-ceramic abutments and all-ceramic crowns (Cercon, Dentsply Sirona Implants) and pre-fabricated standard-shaped (round-diameter) titanium healing abutments followed by final titanium abutments restored with porcelain-fused-to-metal (PFM) implant crowns regarding their biological and functional results when applied in the posterior region.

\section{MATERIALS AND METHODS}

42 patients (19 males and 23 females), with an average age of 48 years (range: 28 - 72 years), were recruited to participate in this randomized controlled trial. The study was approved by the Ethical Committee of the J.W. Goethe University of Frankfurt/Main (registration number 133/05). The study was performed in accordance with the fifth revision of the World Medical Association Declaration of 2000 in Helsinki and the CONSORT statement of 2010. The study was conducted for a 5-year observation period and preliminary results have been obtained after 12-months of loading. Follow up appointments were scheduled at a 3 -month interval for the first year and a 6-month interval for the next 4 years. Participants of the study were informed about the study protocol and gave a written declaration of informed consent. The inclusion criteria were as follows: age of $\geq 18$ years, need for a single posterior implant-supported crown, implant placement exclusively in healed extraction sites, and natural neighboring teeth; no need for extensive augmentation (the simultaneous minor lateral augmentation was accepted) and no parafunctional habits or general contraindications for implant surgery.

Finally, participants of the study had to have the mental and physical ability to enable follow-up over a 5-year period. Patients who had undergone an extended local bone graft procedure in the implant region were excluded. Pregnant women were also excluded. Smoking was not an exclusion criterion.

After inclusion in the study, randomization was performed. Prior to the randomization process, each patient accepted the potential use of both types of prosthetic restoration after implant surgery: zirconia abutments (test group, TG) and titanium abutments (control group, CG). By computer-generated random allocation, each patient was assigned to either the test group or the control group, resulting in a randomized clinical trial with 21 patients (7 males and 14 females), with an average age of 44 years (range: 28 - 68 years) in the test and 21 patients (12 males and 9 females), with an average age of 53 years (range: 34 72 years) in the control group.

Each of the 42 patients received one Ankylos C implant (Dentsply Sirona Implants, York, PA, USA) in the premolar and molar region of the upper and lower jaw. All implants had a diameter of $3.5 \mathrm{~mm}$ and the implant system does not possess an internal index.

Preoperative planning was performed with either panoramic radiographs or cone beam computed tomography (CBCT) and study casts. An hour prior to the operation, a rinse with chlorhexidine solution $(0.2 \%)$ and antibiotic prophylaxis of $2 \mathrm{~g}$ of amoxicillin $(1 \mathrm{~g}$ tablet; or azithromycin in case of allergy) was applied.

The implant placements were performed under local anesthesia (Ultracain with epinephrine $1: 200,000)$ by 2 oral surgeons specialized in implantology, who were both trained with the implant system. After crestal incision, a full thickness flap was raised and osteotomy preparation was conducted according to the protocol of the manufacturer. In each site, implants were inserted subcrestally. If required, minor augmentation procedures (lateral augmentation with xenogenic, bovine-based bone substitute materials (BioOss and resorbable collagen membrane BioGide, Geistlich Biomaterials, Wolhusen, Switzerland)) have been performed simultaneously. Wound closure was performed to achieve submerged healing.

After a healing period of 3 months, the second-stage surgery was performed and, according to the previously performed random allocation, a round-diameter titanium abutment (control group, CG) or an anatomically shaped, 
all-ceramic healing abutment were installed.

Table 1 gives a detailed overview of the implant localization.

In the study group, a prefabricated anatomically shaped, all-ceramic healing abutment (Prototype produced in cooperation with CERCON, Dentsply Sirona Implants) with an oval shape mimicking the natural shape of a molar or premolar tooth has been placed at the second stage surgery. The healing abutments (Fig. 1) correspond to the form and cross-section of a premolar/molar crown and therefore the final abutments (Fig. 2). Two sizes have been applied: a smaller-diameter healing abutment for premolar sites and a larger-diameter one for molar sites. Patients of the control group received a round-diameter standard shaped titanium healing abutment (Ankylos, Dentsply Sirona Implants).

Two weeks after the second-stage surgery, an implantlevel impression was taken using Impregum (3M Espe, St. Paul, MN, USA) in both groups. For each patient, a master cast was fabricated. Depending on the assigned group, either a titanium Balance Posterior abutment (Control group, Dentsply Sirona Implants) or the Ankylos CERCON Balance all-ceramic abutment (Test group, Dentsply Sirona Implants) was placed on the implant analogue. All abutments have been thoroughly investigated by microscopy before insertion to uncover potential micro-cracks. After a 4-week provisional phase with a PMMA (polymethylmethacrylate) crown, the final prosthetic rehabilitation consisting of single all-ceramic crowns (CAD/CAM manufactured yttrium-stabilized zirconium dioxide framework, veneered with glass

Table 1. Detailed overview over allocation to study and control group and implant localization

\begin{tabular}{lcc}
\hline & Control group & Study group \\
\hline Implants in the premolar region & 11 & 13 \\
Implants in the molar region & 10 & 8 \\
\hline
\end{tabular}

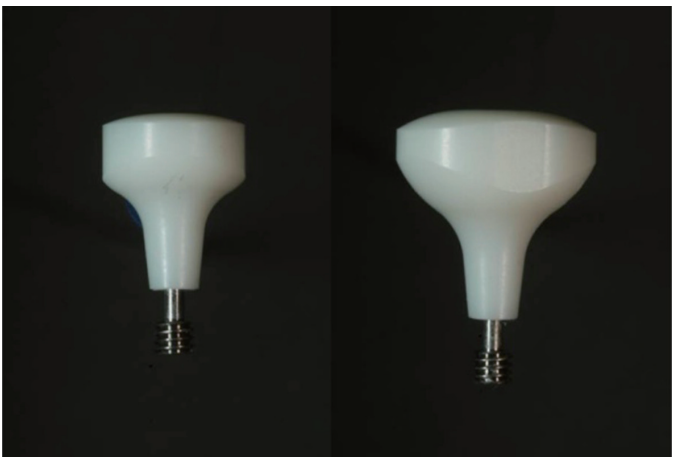

Fig. 1. Prototype zirconia healing abutments were designed and fabricated for the study. The shape mimicked the natural shape of a molar or premolar tooth after crown preparation. ceramics) in the test and single porcelain-fused-to-metal (PFM) implant crowns (CAD/CAM manufactured framework of a chromium-cobalt-molybdenum-alloy (CrKoMo), veneered with glass ceramics) in the control group was performed.

After sandblasting of the titanium abutment with 110 $\mu \mathrm{m}$ aluminium-oxide particles at 2.0 bar, PFM crowns have been cemented with zinc-oxide eugenol provisional cement (TempBond, Kerr dental, Biberach, Germany), while the allceramic crowns were secured with zinc oxide non-eugenol cement (RelyX Temp NE, 3M Espe, St. Paul, MN, USA).

To analyze the influence of healing abutment, definitive abutment, and crown material on peri-implant soft-tissue health and implant success, the following parameters have been evaluated at baseline (after incorporation of final prosthetics) and after 12 months of loading:

- Implant survival

- Peri-implant bone stability

- Implant stability/mobility assessed by Periotest

- The sulcus fluid flow rate (SFRR)

- Plaque index (PI) according to Quigley and Hein ${ }^{14}$

- Technical complications

Orthopantograms recorded at baseline and after 12 months of loading have been analyzed using the Sidexis software program (Sidexis software program, Dentsply Sirona Implants, York, PA, USA) to determine the periimplant bone stability. The distance between the implant shoulder and the first radiographically observable bone-toimplant contact (BIC) was measured at the mesial and distal aspects of the implants to determine the annual bone-level changes. Measurements were conducted by an experienced investigator.

Implant stability/mobility was assessed using the Periotest device (Periotest, Medizintechnik Gulden e.K.). To ensure reliable measurements that allow comparison of obtained data, the widest circumference of the implantretained crown (equator) was used as a reference point for

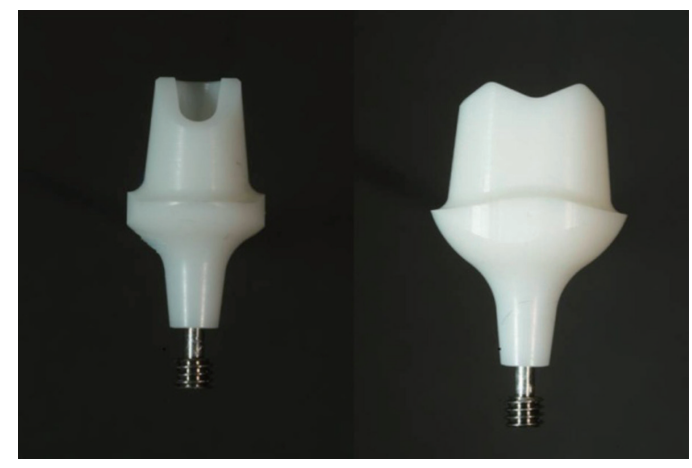

Fig. 2. Final posterior zirconia abutments were also produced. The form and cross-section of the healing and final abutments corresponded perfectly. 
the Periotest device.

The sulcus fluid flow rate (SFFR), measured with the Periotron system (Periotron 8000, Oraflow, Hewlett, NY, USA), and the plaque index (PI) according to Quigley and Hein $^{14}$ were recorded to determine the plaque formation at the different suprastructures and to uncover a potential inflammatory response at the peri-implant soft tissue. The Periotron device records the SFFR as a numerical number, which indicates the health of the peri-implant mucosa. Values from 0 to 10 indicate a healthy mucosa, and values from 10 to 40 indicate a mild peri-implant infection, while values higher than 40 indicate a peri-implant inflammation.

Furthermore, at each follow-up appointment, any technical complications such as loosening or fracture of abutments, chipping of veneering porcelain, and de-cementations were documented in detail.

Intergroup comparisons were conducted with Fisher's exact test. For the quantitative variables, a normality test was first carried out and the data that corresponded with normal distribution were described with mean \pm standard deviations (SDs) and evaluated with Student's t-test or repeated-measures ANOVA. Data that did not correspond with normal distribution were evaluated with Wilcoxon signed-rank test. Differences were deemed significant when the $P$ values were less than $0.05(* P<.05)$ and highly significant when the $P$ values were less than 0.01 (**P $<.01)$.

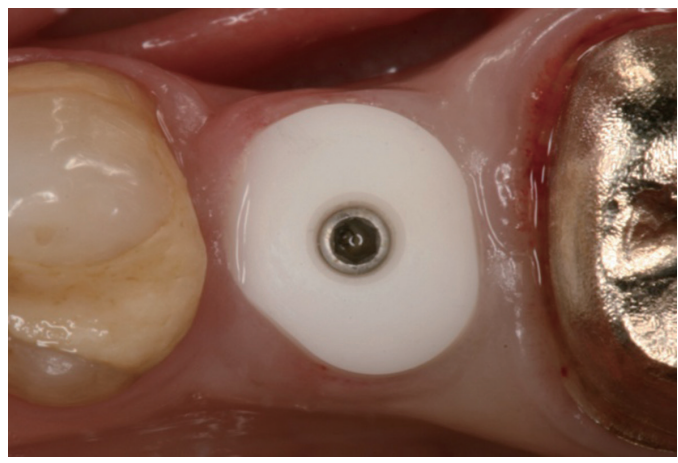

Fig. 3. Soft tissue conditioning and clinically intact attachment to the zirconia healing abutment.

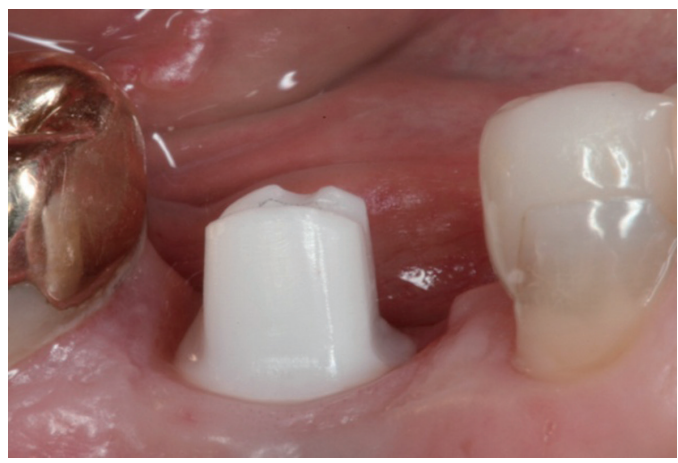

Fig. 5. Zirconia abutment corresponding to the dimension of the healing abutments avoiding any compression of the peri-implant soft tissue.

\section{RESULTS}

In all 42 patients, an uneventful healing after surgical treatment could be observed. Two weeks after incorporation of the prefabricated all-ceramic, anatomically shaped healing abutments and the prefabricated standardshaped (rounddiameter) titanium healing abutments, a naturally dimensioned peri-implant soft tissue without signs of infection could be observed (Fig. 3). When the healing abutment was removed two weeks after the second stage surgery, a clinically intact attachment of the peri-implant gingiva to the healing abutment of the test group was observable (Fig. 4). At the time of the final abutment incorporation, no compression of the soft tissue in the test group could be detected (Fig. 5, Fig. 6). However, the incisions during the secondstage surgery in the TG patients had to be larger than those made in the CG patients, in order to enable mounting of the larger ceramic healing abutments.

Before loading, one ceramic final abutment fracture occurred at the lowest level of the internal cone connection during the try-in procedure, possibly due to incorrect handling by the clinician or technician. This was replaced by a new one before the crown fabrication. From the time of the restoration delivery to the 12-month follow-up appointment, no other major complications developed in either group.

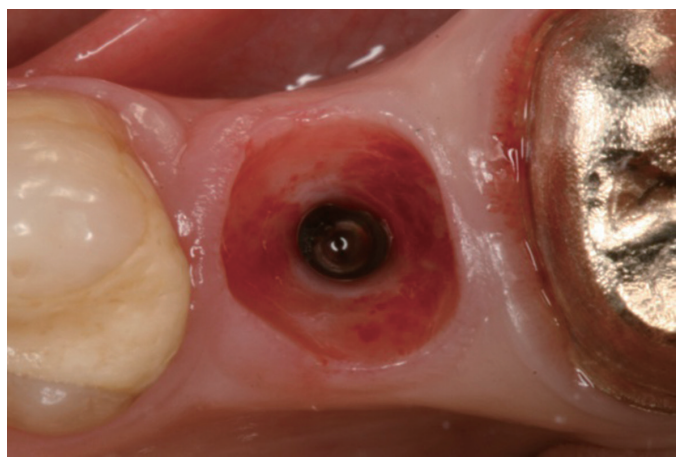

Fig. 4. Preconditioning of the emergence profile two weeks after the mounting of the anatomical shaped.

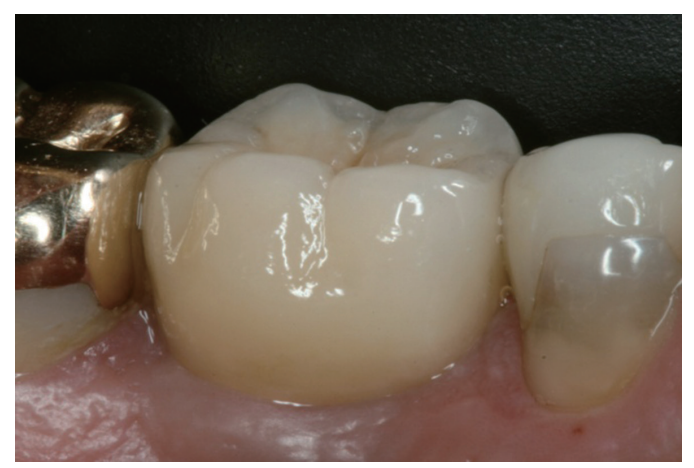

Fig. 6. Final crown in place. 
Minor chipping of the veneering porcelain was observed in 2 patients in the control group at the 12 -month recall appointment and in one patient in the test group at the 6 month recall, resulting in a chipping rate of $9.5 \%$ in the control and $4.8 \%$ in the test group. The difference did not show statistical significance.

The mean $(\mathrm{n}=42)$ sulcus fluid flow rate $\left(\mathrm{mSFFR}_{\text {total }-12}=\right.$ $7.9 \pm 5.4)$ in both groups indicated a healthy peri-implant soft tissue condition at the 12 months follow-up. The difference between the test and the control group at the 12 -months recall $\left(\mathrm{mSFFR}_{\mathrm{ZrO} 2-12}=8.0 \pm 6.2\right.$ mSFFR $_{\mathrm{Ti}-12}=$ $7.9 \pm 4.8$ ) was not significant (Table 2).

The mean $(\mathrm{n}=42)$ plaque index rates $\left(\mathrm{mPI}_{\text {total }-12}=0.4 \pm\right.$ $0.5)$ for both groups at the 12-month recall were generally very low and showed no statistically significant differences between both groups $\left(\mathrm{mPI}_{\mathrm{Ti}-12}=0.3 \pm 0.5\right.$ and $\mathrm{mPI}_{\mathrm{ZrO2}-12}=$ $0.4 \pm 0.7$ ) (Table 2).

Implant stability evaluated with the Periotest device revealed no statistically significant difference between the two groups. The mean Periotest value of all 42 implants revealed $-2.9 \pm 1.9$, while the mean Periotest value of the test group was $-3.2 \pm 1.5$ and that of the control group was $-2.5 \pm 2.3$. This difference was also not statistically significant. Table 2 summarizes the findings of the clinical followup investigation (sulcus fluid flow rate, plaque index rate, and Periotest) at the 12 months follow-up.

Radiological analysis of the peri-implant bone level change after 12 months of loading detected no signs of a peri-implant infection. The mean bone loss (calculated as change between implant shoulder and the most coronal bone-to-implant contact) for the control group was 0.14 $\mathrm{mm}$ mesially and $0.26 \mathrm{~mm}$ distally. In the test group, comparable values of $0.18 \mathrm{~mm}$ mesially and $0.16 \mathrm{~mm}$ distally could be found, but the result was not significant.

\section{DISCUSSION}

In the present study, prefabricated industrially made zirconia healing abutments and subsequent all-ceramic individual abutments and single crowns have been investigated and compared to conventional titanium-based healing and final abutments and PFM single crowns. The anatomically

Table 2. Biological parameters (mean plaque index, mean sulcus fluid rate, and mean Periotest values) in the test (zirconia abutments, $\mathrm{ZrO}_{2}$ ) and in the control group (titanium abutments, Ti)

\begin{tabular}{lccc}
\hline & $\mathrm{mPI}$ & $\mathrm{SFFR}$ & $\mathrm{PTV}$ \\
\hline $\mathrm{ZrO}_{2}$ abutments & $0.4 \pm 0.7$ & $8.0 \pm 6.2$ & $-3.2 \pm 1.5$ \\
Ti abutments & $0.3 \pm 0.5$ & $7.9 \pm 4.8$ & $-2.5 \pm 2.3$ \\
$\begin{array}{l}\text { Significance } \\
\mathrm{ZrO}_{2} \text { vs. Ti }\end{array}$ & $\mathrm{NS}$ & $\mathrm{NS}$ & $\mathrm{NS}$ \\
\hline
\end{tabular}

Statistical significance was set at $P \leq .05$.

NS: not significant shaped zirconia healing abutments have been designed to better shape the peri-implant soft tissue according to the final crown and therefore to replicate the natural tooth contour.

The idea of the present study, to use zirconia ceramic for healing abutments, arise from the low bacterial adherence to zirconia surface shown in different in vitro and in vivo studies. ${ }^{15-17}$ The aim of the study was to investigate if the use of the healing abutment, which usually stays in place over a comparably short period of time, followed by allceramic prosthesis has an impact on the peri-implant soft tissue health compared to the conventional, titanium-based prosthesis.

A clinical benefit of the present approach is that the anatomically shaped healing abutments are not individually produced and are therefore more time-saving and cost-efficient. A major risk factor in the use of zirconia in general requires the excessive machining, which can lead to tensile stresses on the material surface with a direct impact on the material properties. ${ }^{18}$ Because of this, any modifications of these abutments should be kept to a minimum to limit the propagation of this phenomenon.

As the ceramic final abutments have the same shape and cross-section as the ceramic healing abutments used to develop the soft tissue, modifications to the final abutment by the laboratory can be reduced and, if necessary, accomplished very quickly. Additionally, little to no compression of the soft tissue occurs when the zirconia healing abutment is replaced by the final abutment, which makes the clinical procedure more comfortable for the patient, reduces treatment duration, and can reduce or even abandon the need of a prolonged provisional treatment phase.

In both groups, the clinical follow up investigation revealed implant, abutment, and crown survival of $100 \%$, taking in consideration that the single observed zirconia abutment fracture occurred before loading. The chipping of the veneering ceramic that occurred in both groups $(9.5 \%$ in the control and $4.8 \%$ in the test group) is a well-known complication for implant-supported restorations in general and seems, at least in the present study, not to be dependent on the abutment material. ${ }^{19-21}$ However, it is important to state that a proper, anatomically dimensioned framework that offers sufficient support for the veneering ceramic is mandatory to reduce chipping. ${ }^{22}$ In the present study, manufacturing principles of implant-retained crowns have been addressed in both the control and the test group. A thorough analysis of the superstructures with chipping could not identify any trigger factors.

In both groups, implant stability assessed by Periotest revealed successful osseointegration of the implants, but the difference was not significant. No signs of mobility, neither of the implant nor of the abutment-crown complex, could be observed. However, the stability of the implants has to be regarded detached from the abutment or crown material.

Although several studies report a lower bacterial adhesion on zirconia, no significant difference in plaque accumulation could be found in the present study. ${ }^{23-25} \mathrm{~A}$ systematic 
review that evaluated the impact of the abutment characteristics on peri-implant tissue health revealed a significantly less increase in BOP values and plaque accumulation when comparing zirconia and titanium abutments. ${ }^{25}$ From the present study, it has to be noted that all crown margins are set subgingivally and the abutments and crown margins have therefore not been exposed to the oral environment and therefore not accessible to plaque.

Analysis of the sulcus fluid flow rate as an indicator of a potential inflammatory response in the peri-implant soft-tissue detected only a minor, statistically insignificant difference between the test and the control group. This result can be interpreted in contrast to the extensively proven high biocompatibility of zirconia, not only as material for crowns or abutments, but also for dental implants. ${ }^{25,26}$ The literature reveals a high number of publications on all-ceramic abutments and all-ceramic implant-retained single crowns, but the level of evidence, the follow-up period, and the sample size are often inadequate.

In a clinical study, comparing customized zirconia and titanium abutments after 18 months of loading, no statistically significant results regarding survival rates and technical, biologic, and esthetic outcomes could be found. Interestingly, no differences in the color of the peri-implant mucosa was observed, but no additional subdivision according to the gingiva type was performed. ${ }^{27}$

In one of the few long-term follow-up studies on onepiece internal zirconia abutments, 5-year implant survival and occurrence of technical complications were assessed in 52 patients. While the implant survival was $100 \%$ after 5 years of loading, 5 restorations $(7.8 \%)$ had to be remade due to fracture of the internal one-piece zirconia abutment. Four of these fractures occurred in narrow-diameter implants (diameter of $3.3 \mathrm{~mm}$ ), which are known to be a limiting factor for design and construction of one-piece zirconia abutments. ${ }^{28}$

In another long-term study evaluating zirconia abutments restored with all-ceramic crowns in the anterior and premolar region for more than 10 years, no implant or abutment loss could be observed. The cumulative success rate was $96.3 \%$ for abutments and $90.7 \%$ for crowns with technical complications such as screw loosening and minor chipping. After a comparable long observation period, no biological complications or signs of peri-implantitis were observed. ${ }^{29}$

Although all-ceramic abutments and crowns are investigated extensively, no scientific data exist on all-ceramic healing abutments. This might be caused by the fact that individually customized healing abutments from zirconia usually come along with increased cost. The present prefabricated anatomically-shaped healing abutment system might therefore present an interesting method to complete the range of all-ceramic components without additional costs. However, it has to be noted that only few implant systems provide such healing abutments and usually an index-free implant system is required. In this context, a limitation of the present study is that, in the test groups, final abutments were also made from zirconia, and it is therefore difficult to determine the specific influence of the healing abutment on the overall tissue response to the implant prosthetics. Furthermore, the study period of 12 months allows only insights in the short-term tissue reaction.

Within the limitations of the present study, it can be concluded that the use of a prefabricated anatomically shaped all-ceramic healing abutment completes the range of all-ceramic components and can reduce treatment duration, discomfort, and need of shaping the peri-implant soft-tissue with successive provisional devices. Regarding the analysis of implant survival, implant stability, and peri-implant soft tissue response, no statistically significant differences between the two groups could be found.

\section{CONCLUSION}

The preliminary results regarding the biological stability and technical behaviour of all-ceramic restorations compared to conventional, titanium-based implant prosthetics in posterior regions showed no statistically significant superiority of the all-ceramic procedure. However, the use of all-ceramic components, including prefabricated all-ceramic healing abutments, might present an encouraging treatment option in times of rising demand for metal-free dental implant prosthetics. Larger sample-size clinical trials with a longer follow-up period and study designs investigating the particular benefit of all-ceramic healing abutments are necessary to further increase the tissue-compatibility of implantretained prosthetics.

\section{ORCID}

Paul Weigl https://orcid.org/0000-0001-7434-7988

Jonas Lorenz https://orcid.org/0000-0002-4209-3992

\section{REFERENCES}

1. Linkevicius T, Puisys A, Linkeviciene L, Peciuliene V, Schlee M. Crestal bone stability around implants with horizontally matching connection after soft tissue thickening: A prospective clinical trial. Clin Implant Dent Relat Res 2015;17:497508.

2. Linkevicius T, Puisys A, Steigmann M, Vindasiute E, Linkeviciene L. Influence of vertical soft tissue thickness on crestal bone changes around implants with platform switching: A comparative clinical study. Clin Implant Dent Relat Res 2015;17:1228-36.

3. Bassetti RG, Stähli A, Bassetti MA, Sculean A. Soft tissue augmentation around osseointegrated and uncovered dental implants: a systematic review. Clin Oral Investig 2017;21:5370 .

4. Lewis MB, Klineberg I. Prosthodontic considerations designed to optimize outcomes for single-tooth implants. A review of the literature. Aust Dent J 2011;56:181-92.

5. Furze D, Byrne A, Alam S, Wittneben JG. Esthetic outcome of implant supported crowns with and without peri-implant 
conditioning using provisional fixed prosthesis: A randomized controlled clinical trial. Clin Implant Dent Relat Res 2016;18:1153-62.

6. Esposito M, Bressan E, Grusovin MG, D’Avenia F, Neumann K, Sbricoli L, Luongo G. Do repeated changes of abutments have any influence on the stability of peri-implant tissues? One-year post-loading results from a multicentre randomised controlled trial. Eur J Oral Implantol 2017;10:57-72.

7. Alshhrani WM, Al Amri MD. Customized CAD-CAM healing abutment for delayed loaded implants. J Prosthet Dent 2016;116:176-9.

8. Wittneben JG, Buser D, Belser UC, Brägger U. Peri-implant soft tissue conditioning with provisional restorations in the esthetic zone: the dynamic compression technique. Int J Periodontics Restorative Dent 2013;33:447-55.

9. Lops D, Bressan E, Parpaiola A, Sbricoli L, Cecchinato D, Romeo E. Soft tissues stability of cad-cam and stock abutments in anterior regions: 2-year prospective multicentric cohort study. Clin Oral Implants Res 2015;26:1436-42.

10. Staubli N, Walter C, Schmidt JC, Weiger R, Zitzmann NU. Excess cement and the risk of peri-implant disease - a systematic review. Clin Oral Implants Res 2017;28:1278-90.

11. Linkevicius $T$, Vaitelis $J$. The effect of zirconia or titanium as abutment material on soft peri-implant tissues: a systematic review and meta-analysis. Clin Oral Implants Res 2015;26: 139-47.

12. Nothdurft FP, Nonhoff J, Pospiech PR. Pre-fabricated zirconium dioxide implant abutments for single-tooth replacement in the posterior region: success and failure after 3 years of function. Acta Odontol Scand 2014;72:392-400.

13. Ekfeldt A, Fürst B, Carlsson GE. Zirconia abutments for single-tooth implant restorations: a retrospective and clinical follow-up study. Clin Oral Implants Res 2011;22:1308-14.

14. Quigley GA, Hein JW. Comparative cleansing efficiency of manual and power brushing. J Am Dent Assoc 1962;65:26-9.

15. Scarano A, Piattelli M, Caputi S, Favero GA, Piattelli A. Bacterial adhesion on commercially pure titanium and zirconium oxide disks: an in vivo human study. J Periodontol 2004; 75:292-6.

16. Welander M, Abrahamsson I, Berglundh T. The mucosal barrier at implant abutments of different materials. Clin Oral Implants Res 2008;19:635-41.

17. Pabst AM, Walter C, Bell A, Weyhrauch M, Schmidtmann I, Scheller H, Lehmann KM. Influence of CAD/CAM zirconia for implant-abutment manufacturing on gingival fibroblasts and oral keratinocytes. Clin Oral Investig 2016;20:1101-8.

18. Sundh A, Molin M, Sjögren G. Fracture resistance of yttrium oxide partially-stabilized zirconia all-ceramic bridges after veneering and mechanical fatigue testing. Dent Mater 2005;21: 476-82.

19. Zembic A, Sailer I, Jung RE, Hämmerle CH. Randomizedcontrolled clinical trial of customized zirconia and titanium implant abutments for single-tooth implants in canine and posterior regions: 3-year results. Clin Oral Implants Res 2009; 20:802-8.

20. Sailer I, Zembic A, Jung RE, Siegenthaler D, Holderegger C, Hämmerle $\mathrm{CH}$. Randomized controlled clinical trial of cus- tomized zirconia and titanium implant abutments for canine and posterior single-tooth implant reconstructions: preliminary results at 1 year of function. Clin Oral Implants Res 2009;20:219-25.

21. Brägger U, Karoussis I, Persson R, Pjetursson B, Salvi G, Lang N. Technical and biological complications/failures with single crowns and fixed partial dentures on implants: a 10-year prospective cohort study. Clin Oral Implants Res 2005;16:326-34.

22. Jung RE, Pjetursson BE, Glauser R, Zembic A, Zwahlen M, Lang NP. A systematic review of the 5-year survival and complication rates of implant-supported single crowns. Clin Oral Implants Res 2008;19:119-30.

23. Scarano A, Piattelli M, Caputi S, Favero GA, Piattelli A. Bacterial adhesion on commercially pure titanium and zirconium oxide disks: an in vivo human study. J Periodontol 2004; 75:292-6.

24. Rimondini L, Cerroni L, Carrassi A, Torricelli P. Bacterial colonization of zirconia ceramic surfaces: an in vitro and in vivo study. Int J Oral Maxillofac Implants 2002;17:793-8.

25. Sanz-Martín I, Sanz-Sánchez I, Carrillo de Albornoz A, Figuero E, Sanz M. Effects of modified abutment characteristics on peri-implant soft tissue health: A systematic review and meta-analysis. Clin Oral Implants Res 2018;29:118-29.

26. Holländer J, Lorenz J, Stübinger S, Hölscher W, Heidemann D, Ghanaati S, Sader R. Zirconia dental implants: Investigation of clinical parameters, patient satisfaction, and microbial contamination. Int J Oral Maxillofac Implants 2016;31:855-64.

27. Bösch A, Jung RE, Sailer I, Goran B, Hämmerle CH, Thoma DS. Single-tooth replacement using dental implants supporting all-ceramic and metal-based reconstructions: Results at 18 months of loading. Int J Periodont Restor Dent 2018;38:1739.

28. Nilsson A, Johansson LÅ, Lindh C, Ekfeldt A. One-piece internal zirconia abutments for single-tooth restorations on narrow and regular diameter implants: A 5-year prospective follow-up study. Clin Implant Dent Relat Res 2017;19:916-25.

29. Zembic A, Philipp AO, Hämmerle CH, Wohlwend A, Sailer I. Eleven-year follow-up of a prospective study of zirconia implant abutments supporting single all-ceramic crowns in anterior and premolar regions. Clin Implant Dent Relat Res 2015;17:e417-26. 